\title{
Lichen atrophicus und Vitiligo.
}

\author{
Von \\ W. Orbaek, \\ ehem. Assistent der dermatol. Poliklinik Kopenhagens.
}

Seit E. Wils on im Jahre 1869 die Krankheit beschrieb, welche er Lichen planus nannte, ist viel über diese Krankheit in der modernen Dermatologie gestritten worden. Es ist hier nicht der Ort den gegenwärtigen Stand der Lichenfrage zu discutiren. Nur soviel soll gesagt werden, dass der Wils o n'sche Lichentypus (Lichen ruber planus) scharf ${ }^{\circ}$ von dem HebraKaposi'schen L. r. acuminatus getrennt werden muss.

Halten wir uns jetzt an die ersten dieser zwei Typen, kann man um diese als Centrum einige Varietäten gruppiren, welche von verschiedenen Autoren beschrieben sind, z. B. L. obtusus, L. corneus ( $\mathrm{Vidal}$ ), L. c. hypertrophicus, je nach der verschiedenen Grösse der papulösen Elemente.

Die epitheliale Verdickung ist eine bekanntlich consecutive, Lichen ist weder eine Keratose, noch ein Hautcatarrh. Die Krankheit beginnt mit einer Zellinfiltration der oberen Schichten des Coriums. Man findet jedoch eine Varietät, bei welcher die Atrophie gleich von Anfang der Krankheit iiberwiegend ist. Hallopeau ist der erste, der diese Form genau beschrieben hat, und er nennt sie Lichen atrophicus (seu sclerosus); Kaposi erwähnt kurz in seinem Handbuch einen Fall ron, wie es scheint, dieser Affection, aber er meint, dass es sich um ein anormales Terminalstadium von Lichen planus handelt. Wir geben hier einige bibliographische Noten über diese ziemlich seltene Affection.

Wie erwähnt, ist Hallopea u der erste, der diese Form genau beschrieb. In einer Vorlesung im Jahre 1887: „Du lichen planus et particulièrement de sa forme atrophique" zeigt er, dass die papulösen Elemente nicht so stark gefärbt sind 
wie die der gewöhnlichen Form des Lichen. Sie bilden schnell weisse, narbenähnliche Flecke mit vielen feinen, punktförmigen Depressionen. Es handle sich um eine Jocale cutane Sclerose, ohne jedoch eine Scleroderme en plaque (morphie) zu sein; mikroskopisch sieht man die Epidermis verdickt, die Coriumpapillen beinahe verschwunden. Im Jahre 1889 berichtet derselbe Autor über seinen zweiten Fall, er erwähnt jetzt, dass es sich um eine eigenthümliche Form von Lichen handelt, nicht um ein Terminalstadium. Man sehe die atrophischen, narbenähnlichen Flecke gleich zu Beginn der Krankheit, er schlägt deshalb den Namen Lichen sclerosus vor.

Im Jahre 1892 untersucht Darier den dritten Fall; die Affection ist hier 2 Jahre alt; die narbenähnlichen weissen Flecke sind von einer rosa Zone umgeben, sie sind parallel mit den Falten der Haut, von Erbsen- bis Mandelgrösse, gruppirt "en mosaique"; an den Flecken findet man viele kleine, gelbe "points cornés". Die Epidermis ist stark verdickt, die kleinen gelben Punkte dringen wie kleine Zapfen in die Haarfollikeln, das Strat. Malpighi ist normal. Die Papillen und die interpapillären Zapfen sind sehr klein, der juxta-papilläre Theil des Coriums sclerosirt. Gland. sudorip und Haarbulbi normal.

Pawlow bespricht 1894 in der russischen dermatologischen Gesellschaft einen Fall von L. atrophic., an dessen weissen Flecken er schwarze, comedonenähnliche Punkte bemerkt; er gibt keine nähere Beschreibung oder Mikroskopie.

Hallopeau erwähnt ähnliche schwarze Punkte als ein Anfangsphänomen im Jahre 1896, aber meint doch, dass die kleinen Depressionen die Hauptsache für die Diagnose sind.

Im November 1898 stellt endlich H. den letztbeschriebenen Fall von L. atroph. in der Société de Dermatologie vor. Die Affection ist hier 2 Jahre alt, ganz ähnlich der oben beschriebenen, die Flecke finden sich besonders zwischen der Mamma und confluiren hier (Lichen en nappe). Die Grösse der Flecken variirt in allen diesen Fällen von Erbsengrösse bis $45 \times 32 \mathrm{Cm}$. ; die Eruption ist stark juckend, von allen Patienten wird mitgetheilt, dass sie ron nervösem Temperament waren. Die Dauer der Krankheit schwankt von einigen Monaten bis zu 8 Jahren.

Ich erlaube mir einen Fall mitzutheilen, welchen ich in meiner Privatpraxis beobachtet und beinahe 1 Jahr behandelt habe. 
Pat. ist ein 23jähriger Mann, kräftig gebaut. Gewicht: 70 Kilo, Länge: $165 \mathrm{Cm}$., er ist der jüngste ron $6 \mathrm{Geschwistern.} \mathrm{Die} \mathrm{Mutter} \mathrm{leidet}$ seit mehreren Jahren an Diabetes mellitus, Vater gesund, von den Schwestern findet man bei einer Zeichen von Hysteria (Aphonie, Convulsionen), eine andere Schwester hat Urticaria ("autographismus"). Der Pat. ist immer gesund gewesen, er ist intelligent, sehr lebhaft. Kein Alkoholismus, keine venerischen Krankheiten. Vor 2 Jahren litt Pat. an einer rechtsseitigen Ischias, wie es scheint nach Erkältung.

Die Hautaffecte, für welche Pat. Behandlung sucht, meint er vor ca. 10 Jahren bemerkt zu haben, er ist zufällig darauf aufmerksam geworden als Knabe beim Schwimmen. Er hat immer Jucken bemerkt, zeitweise auch Parästhesien, besonders ein Gefühl wie Uebergiessen der Partien mit warmem Wasser.

Die Regio trochanterica sin. ist in einer Ausdehnung von ca. $1 \frac{1}{2}$ Handteller bedeckt mit zahlreichen, theils isolirten, theils confluirten narbenähnlichen Flecken. - Die Grösse variirt, man findet confluirte Plaques von $8 \mathrm{~cm}$. Diameter. Die Fleeken sind leicht deprimirt, ihre Epidermis ist schimmernd grau-rosa, mit grauen Streifen und man sieht eine Menge nadelstichgrosser Vertiefungen, eine centrale Vertiefung ist besonders deutlich markirt. Die Epidermis ist von der Consistenz von Pergamentpapier, keine Abschälung. Jeder Fleck ist von einer $1 / 2 \mathrm{Cm}$. breiten, sepia-gefärbten Zone umgeben, welche sehr fein, aber deutlich radiär gestreift ist. Bei Confluenz mehrerer Flecke entstehen kreis- und festonähnliche Figuren; die Entwicklung schreitet centrifugal weiter. In der Reg. inguinal. dxt. findet man 4-5 Flecke, jeden von $3 \mathrm{Cm}$. Diameter. Im Laufe der letzten 2-3 Monate hatten sich am l. Unterarm, nahe dem Handgelenke, mehrere kleine Flecke entwickelt, welche gleich vom Anfang, auch als sie nur mit der Lupe sichtbar waren, dasselbe atrophische, leicht excoriirte Aussehen zeigten, sie entwickelten sich äusserst langsam.

Die Affection ist, wie gesagt, von starkem Jucken begleitet, der Schlaf ist oft gestört.

Am Genitale und in der Regio inguinalis findet sich eine cutane Dyschromie, welche besonders stark an der Pars pendula penis und in der Regio pubica ausgeprägt ist. An diesen Stellen finden sich mehrere $2 \mathrm{Cm}$. grosse Flecke, welche ganz pigmentfrei sind. Die Haut der Reg. pubica ist gelbweiss, die Haare normal, die pigmentfreien Stellen überschreiten nicht die Haargrenze. Keine Adenitis.

Die Sensibilität ist normal, nur findet sich ein wenig Hyperästhesie für Nadelstiche über der Columna; Temperatursinn auch an den ergriffenen Partien normal, keine Anästhesie.

In den letzten 2 Jahren fühlt Pat. anfallsweise Schmerzen in der r. Kopfhälfte, der Anfall beginnt mit subjectiven Lichtempfindungen, und dazu kommen später Sensibilitätsanomalien in p. Arm und Hand, besonders Anästhesie für kleine Gegenstände (Feder, Nadel). Der Anfall dauert einige Stunden, ist von Schläfrigkeit begleitet. Niemals Convulsionen, noch Bewusstlosigkeit. Die Schleimhäute normal gefärbt, Urin ohne fremde Bestandtheile.

Dieser Fall ist ein typisches Beispiel der atrophischen Form der Lichen, wir finden alle, von den verschiedenen Autoren beschriebenen Stigmata - besonders die punktförmigen Depressionen und die Gruppirung der Elemente „en mosaique“, welche namentlich Fournier pointirt. Das Jucken ist hier sehr stark gewesen, seit mehreren Jahren constant und ist zusammen mit den Parästhesien sehr quälend für den Patienten; 
Page gibt - nach Selbstbeobachtung - eine genaue Beschreibung des Juckens bei Lichen, welches jenem, das Pat. gefühlt hat, $z u$ entsprechen scheint.

Die von Ziemsen, Brocq, Hardy und Wickham beschriebenen grauen Streifen sind hier sehr deutlich. Entscheidend für die Diagnose dieses Falles ist das Aussehen der Flecken und ihr atrophischer Charakter, welchen man hier gleich von Anfang constatiren kann. Dies liefert den Beweis, dass es sich nicht um ein Terminalstadium handelt, sondern dass hier eine von Anfang an eigenthümliche Form vorliegt.

Die mikroskopische Untersuchung eines von der Reg. troch. excidirten Stückes zeigte nur starke Epithel-Verdickung, mit deutlichen in die Haarfollikel reichenden Zapfen (punktförmige Depression), die Papillen des Corium nur als eine undeutliche Bogenlinie sichtbar.

Bezüglich der Aetiologie des Lichen - speciell der atrophischen Form - sind bekanntlich die Meinungen verschieden.

Hallopeau meinte im Jahre 1896 bei der Demonstration seines Falles, dass die Krankheit eine parasitäre sei und glaubt, dass der Parasit in der Drüsenmündung gesucht werden müsse. Wir haben deshalb Inoculationen am Patienten versucht, aber ohne Erfolg.

Wir meinen, dass im oben citirten Falle die Licheneruption nicht von den Vitiligoflecken getrennt werden kann. Wel a n d e r hat im Jahre 1894 eine sehr interessante Krankengeschichte berichtet: Bei einem 24jähr. Manne sind nach einer beftigen Gemüthserschütterung Symptome von Lichen planus, Vitiligo und Neurodermitis circumscripta aufgetreten. Wir meinen, dass dieser Fall einige Aehnlichkeit mit dem unsrigen hat. Die meisten Autoren meinen, dass es sich bei Vitiligo um ein nervöses Moment handelt; sei es um periphere Neuritiden (Leloir, Schwimmer), oder um eine Folge verschiedener Krankheiten (Besnier).

Die Therapie ist in unserem Falle theils eine locale Pyrogallussalbe, - theils eine generelle - Arsen - gewesen. Die locale hat uns ganz gute Resultate gegeben - die generelle, anfangs alle in angewendet, scheint selbt bei längerer Dauer ohne Werth zu sein. 